\title{
Effects of three-dimensionally printed polycaprolactone $/ \beta$-tricalcium phosphate scaffold on osteogenic differentiation of adipose tissue- and bone marrow-derived stem cells
}

\author{
Hannara Park ${ }^{1}$, \\ Jin Soo Kim ${ }^{1}$, \\ Eun Jung $\mathrm{Oh}^{2}$, \\ Tae Jung Kim², \\ Hyun Mi Kim², \\ Jin Hyung Shim ${ }^{3}$, \\ Won Soo Yoon ${ }^{3}$, \\ Jung Bo Huh", \\ Sung Hwan Moon ${ }^{5}$, \\ Seong Soo Kang ${ }^{6}$, \\ Ho Yun Chung ${ }^{2}$ \\ ${ }^{1}$ Department of Plastic and \\ Reconstructive Surgery, Daegu Fatima \\ Hospital, Daegu; \\ ${ }^{2}$ Department of Plastic and \\ Reconstructive Surgery, School of \\ Medicine, Kyungpook National \\ University, Daegu; \\ ${ }^{3}$ Department of Mechanical Engineering, \\ Korea Polytechnic University, Siheung; \\ ${ }^{4}$ Department of Prosthodontics, Dental \\ Research Institute, Institute of \\ Translational Dental Science, School of \\ Dentistry, Pusan National University, \\ Yangsan; \\ ${ }^{5}$ Department of Medicine, Konkuk \\ University School of Medicine, Seoul; \\ ${ }^{6}$ College of Veterinary Medicine, \\ Chonnam National University, \\ Gwangju, Korea
}

\begin{abstract}
Background: Autogenous bone grafts have several limitations including donor-site problems and insufficient bone volume. To address these limitations, research on bone regeneration is being conducted actively. In this study, we investigate the effects of a three-dimensionally (3D) printed polycaprolactone (PCL)/tricalcium phosphate (TCP) scaffold on the osteogenic differentiation potential of adipose tissue-derived stem cells (ADSCs) and bone marrow-derived stem cells (BMSCs). Methods: We investigated the extent of osteogenic differentiation on the first and tenth day and fourth week after cell culture. Cytotoxicity of the 3D printed PCL/ $\beta$-TCP scaffold was evaluated by 3-(4,5-dimethylthiazol-2-yl)-5-(3-carboxymethoxyphenyl)-2-(4-sulfophenyl)-2H-tetrazolium assay, prior to osteogenic differentiation analysis. ADSCs and BMSCs were divided into three groups: $C$, only cultured cells; $M$, cells cultured in the 3D printed PCL/ $\beta$-TCP scaffold; D, cells cultured in the $3 \mathrm{D}$ printed PCL/ $\beta$-TCP scaffold with a bone differentiation medium. Alkaline phosphatase (ALP) activity assay, von Kossa staining, reverse transcription-polymerase chain reaction (RT-PCR), and Western blotting were performed for comparative analysis.

Results: ALP assay and von Kossa staining revealed that group $M$ had higher levels of osteogenic differentiation compared to group C. RT-PCR showed that gene expression was higher in group M than in group $C$, indicating that, compared to group $C$, osteogenic differentiation was more extensive in group $M$. Expression levels of proteins involved in ossification were higher in group $M$, as per the Western blotting results.

Conclusion: Osteogenic differentiation was increased in mesenchymal stromal cells (MSCs) cultured in the 3D printed PCL/TCP scaffold compared to the control group. Osteogenic differentiation activity of MSCs cultured in the 3D printed PCL/TCP scaffold was lower than that of cells cultured on the scaffold in bone differentiation medium. Collectively, these results indicate that the $3 \mathrm{D}$ printed PCL/TCP scaffold promoted osteogenic differentiation of MSCs and may be widely used for bone tissue engineering.
\end{abstract}

Keywords: Polycaprolactone / Tricalcium phosphate / Adipose tissue / Bone marrow / Stem cells / Cell differentiation / Mesenchymal stromal cells / Tissue engineering
Correspondence: Ho Yun Chung

Department of Plastic and Reconstructive Surgery, School of Medicine, Kyungpook

National University, 680 Gukchaebosang-ro, Jung-gu, Daegu 41944, Korea

E-mail: hy-chung@knu.ac.kr

${ }^{\star}$ This article was presented at the 8th R \& R Forum on April 19, 2018 in Jeonju, Korea.

Received April 16, 2018 / Revised August 13, 2018 / Accepted September 10, 2018

\section{INTRODUCTION}

Autologous bone grafts are used in reconstructive surgery for congenital malformations and defects caused by trauma or a 
disease, as well as in cosmetic procedures for certain parts of the body. However, they have disadvantages such as limited donor sites, discomfort, and donor site morbidity. Therefore, extensive research has been conducted to develop synthetic bone graft materials. However, allografts or artificial bone substitutes can act as foreign bodies following their implantation in the human body, and induce problems including inflammation and implant rejection. Accordingly, considerable efforts have been made to develop materials that can minimize these drawbacks [1].

Mesenchymal stromal cells (MSCs) have been known as a cell population that exhibits pluripotent capabilities for 40 years, and they are still actively studied because they may be utilized in the biosynthesis of multiple body organs [2]. MSCs have been widely used in studies of synthetic bone graft materials. MSCs do not have high osteogenic efficiency on their own and do not reflect the inherent properties of bone tissue. Therefore, researchers have been culturing MSCs with various synthetic biomaterials to create adequate synthetic bone graft materials.

Scaffolds are synthetic biomaterials with mesh, foam, predesigned structures that are used to increase cell attachment, proliferation, and tissue formation. An ideal scaffold must have biocompatibility, biodegradability, and good mechanical strength to support the defective area. Furthermore, it must have suitable porosity, pore size, and interconnected network for cell penetration, and is considered even better if it can provide calcium and phosphorus at adequate ratios [3].

Numerous methods have been used to produce fabricating scaffolds. It is difficult to obtain the desired pore size using conventional methods such as solvent casting, fiber meshes, melt molding, and freeze drying. Although methods using organic solvents have been developed to overcome this limitation, these organic solvents can induce host reactions such as inflammation and toxicity. Recently, methods of producing scaffolds using rapid prototype manufacturing techniques have been highlighted [4]. They include selective laser-sintering (SLS), threedimensional (3D) printing, extrusion technology-based systems, solid grounding curing, and stereolithography apparatus (SLA). In this study, fabricating scaffolds were produced using an easily performed 3D printing technology process, which can be used in ambient environments.

Among the various biomaterials used to prepare scaffolds, polycaprolactone (PCL) is a biodegradable polyester material that has excellent electro-spinnability, good mechanical features, good biocompatibility, and low immunogenicity. These qualities make it one of the most attractive tissue regenerative materials [5]. PCL is biodegradable and completely degrades 3-4 years after transplantation inside the body, and it has excellent stability and osteoconduction affinity [6].
Tricalcium phosphate (TCP) is a bioabsorbable ceramic that is rapidly degraded into calcium and phosphate in the body. Its absorption rate can be controlled by combining it with hydroxyapatite, which has low solubility. TCP loses its mechanical strength over time, which can lead to an implant failure if the mechanical strength of the cells does not increase as the mechanical strength of TCP decreases. However, the double holes on the outer and inner TCP surfaces allow vascular or bone tissue to penetrate the graft material following its implantation inside the body. Therefore, TCP has been used in various implantation procedures. It is also used to induce rapid and stable osseointegration at bony defects or in the cranium [7]. There are two forms of TCP, $\alpha$-TCP, and $\beta$-TCP, which both have a similar solubility, but differ in their thermodynamic stability in the biological environment and at normal temperature ranges [8]. Although the biodegradation of both forms is affected by a number of external factors such as surface area, crystallinity, crystal perfection, and ionic substitutions, $\alpha$-TCP is more rapidly biodegraded than $\beta$-TCP [9].

Recently, research on bone tissue engineering using MSCs and various synthetic biomaterials has been actively conducted. However, no study has investigated whether a PCL/ $\beta$-TCP scaffold fabricated using $3 \mathrm{D}$ printing has any effects on osteogenic differentiation. Therefore, the present study investigated the effects of the 3D printed PCL/ $\beta$-TCP scaffold, which has specific biochemical properties and excellent biocompatibility, on the osteogenic differentiation of adipose tissue-derived stem cells (ADSCs) and bone marrow-derived stem cells (BMSCs). In addition, we determine whether or not the scaffold induces osteogenic differentiation in the absence of a bone differentiation medium.

\section{METHODS}

Two experiments were conducted to investigate the effects of the $3 \mathrm{D}$ printed PCL/ $\beta$-TCP scaffold on ADSCs and BMSCs. Before conducting the experiment on the effects of the $3 \mathrm{D}$ printed PLC/ $\beta$-TCP scaffold on the osteogenic differentiation of ADSCs and BMSCs, a 3-(4,5-dimethylthiazol-2-yl)-5-(3carboxymethoxyphenyl)-2-(4-sulfophenyl)-2H-tetrazolium (MTS) assay was performed to evaluate the cytotoxicity of the scaffold and confirm its safety for MSC culture.

\section{Fabrication of 3D printed PCL/ $\beta$-TCP scaffolds}

Biodegradable PCL (Evonik Industries, Essen, Germany) and TCP (Sigma-Aldrich, St. Louis, MO, USA) were used as materials for the scaffolds. PCL was dried at $105^{\circ} \mathrm{C}$ for 1 day before use. $\beta$-TCP had a particle size of $100 \mathrm{~nm}$ or less. The $3 \mathrm{D}$ printed 
PCL/ $\beta$-TCP scaffolds were made in a heating jacket and stainless steel cylinder with a micro-nozzle along an X-Y-Z axis. The scaffolds were designed and fabricated using in-house CAD/ CAM software. A steel syringe containing the PCL $/ \beta$-TCP mixture was equipped to a $3 \mathrm{D}$ printer and maintained at $120^{\circ} \mathrm{C}$. The molten PCL/ $\beta$-TCP mixture was precisely dispensed through stainless steel nozzle with diameter of $300 \mu \mathrm{m}$.

\section{Biological safety of $3 \mathrm{D}$ printed PCL/ $\beta$-TCP scaffold cultured with MSCs}

MTS assay of the 3D printed PCL/ $\beta$-TCP scaffold: the ADSCs and BMSCs were loaded into a 96-well plate at a density of $1 \times 10^{5}$ cells/well. Each cell culture group and the cell group cultured in the scaffold were incubated at $37^{\circ} \mathrm{C}$ exposed to $5 \% \mathrm{CO}_{2}$ in an incubator for 24 hours and 4 weeks, respectively. The medium was removed and then $100 \mu \mathrm{L}$ Dulbecco's modified Eagle's medium (Gibco, Paisley, Scotland) and $20 \mu \mathrm{L}$ MTS reagent (Promega G3582, Madison, WI, USA) were added, and the cells were cultured for 4 hours in the incubator. Then, the absorbance of the cell suspension was measured at a $490 \mathrm{~nm}$ using an enzyme-linked immunosorbent assay plate reader. The scaffold toxicity was measured and expressed relative to the control measurement.

\section{Comparison of osteogenic differentiation of MSCs in the presence of $3 \mathrm{D}$ printed $\mathrm{PCL} / \beta$-TCP scaffold}

An in vitro experiment was performed to study the osteogenic activity of ADSCs and BMSCs. Levels of osteogenic differentia- tion were measured on day 1 and 10 and week 4 after cell culturing (Fig. 1). The ADSCs and BMSCs were divided into the following three groups for the experiment: $\mathrm{C}$, only cultured cells; $\mathrm{M}$, cells cultured in the $3 \mathrm{D}$ printed PCL/ $\beta$-TCP scaffold; and $\mathrm{D}$, cells cultured in the $3 \mathrm{D}$ printed PCL/ $\beta$-TCP scaffold with a bone differentiation medium. Alkaline phosphatase (ALP) assay, von Kossa staining, reverse transcription-polymerase chain reaction (RT-PCR), and Western blotting were used to analyze and compare the levels of osteogenic differentiation of the three groups depending on the time point of the culture period. The ALP assay results were obtained on day 1 and at 10 after cell culture. The von Kossa staining results were obtained 4 weeks after cell culturing while the RT-PCR and Western blotting results were obtained on days 1 and 10 and week 4 after cell culture.

\section{Reverse transcription-polymerase chain reaction}

Total RNA was isolated using Trizol (Thermo Fisher Scientific, Waltham, MA, USA) according to the manufacturer's instructions. cDNA was prepared using the RevertAid first strand cDNA synthesis kit (Thermo Fisher Scientific) and RT-PCR was performed using the AccuPower RT-PCR kit (Bioneer, Daejeon, Korea). To normalize the input load of cDNA among the samples, glyceraldehyde-3-phosphate dehydrogenase $(G A P D H)$ was used as an endogenous standard. Type I collagen $(C O L 1)$, osteocalcin $(O C N)$, runt-related transcription factor 2 (RUNX2), and GAPDH expression levels were compared using RT-PCR (Table 1).

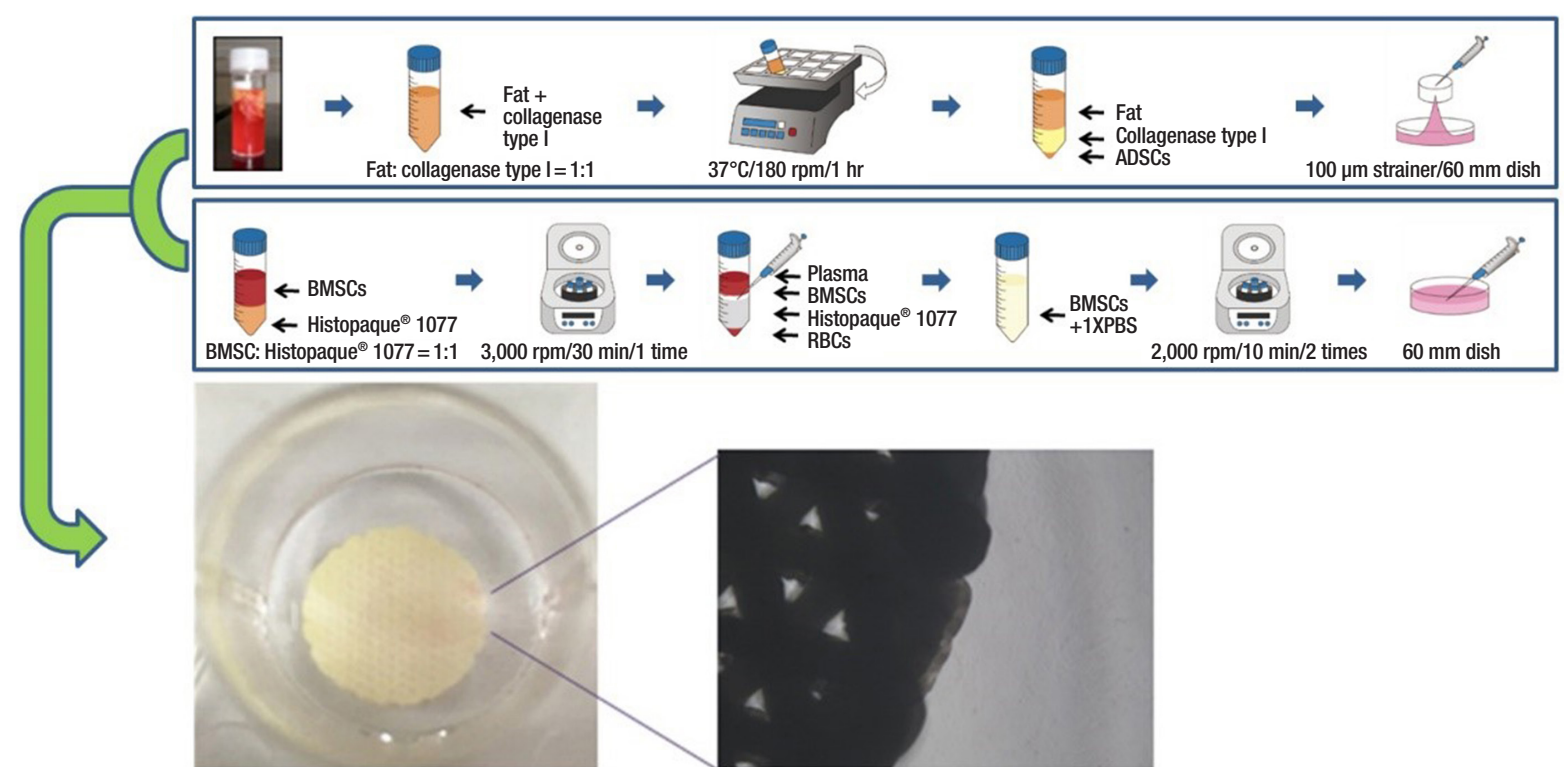

Fig. 1. Collection of adipose tissue-derived stem cells (ADSCs) and bone marrow-derived stem cells (BMSCs), and seeding inside threedimensionally (3D) printed polycaprolactone (PCL)/ $\beta$-tricalcium phosphate (TCP) scaffold. RBC, red blood cell; PBS, phosphate-buffered saline. 


\section{Statistical analysis}

Statistical analysis was assessed with SPSS ver. 22.0 (IBM Corp., Armonk, NY, USA). Subgroup analysis was performed with the paired $t$-test. Statistical significance was set at $p<0.05$.

\section{RESULTS}

Safety analysis of interaction between 3D printed PCL/ $\beta$-TCP scaffold and MSCs

No significant difference was observed between the 3D printed

Table 1. Primer sequences and reaction conditions for reverse transcription-polymerase chain reaction

\begin{tabular}{lllc}
\hline Gene & & \multicolumn{1}{c}{ Sequence $\left(5^{\prime} \rightarrow 3\right)$} & Reaction condition \\
\hline COL1 & Forward & TAGGGTCTAGACATGTTCAGCTTTGT & Denaturation step: $94^{\circ} \mathrm{C}$ \\
& Reverse & GTGATTGGGGGATGTCTCGT & for 45 seconds \\
OCN & Forward & CCATGAGGACCCTCTCTCTG & Annealing step: $62^{\circ} \mathrm{C}$ for \\
& Reverse & AAGCAGGGTCAAGCTCACAT & 60 seconds \\
RUNX2 & Forward & CGCATTCCTCATCCCAGTAT & Extension step: $72^{\circ} \mathrm{C}$ for \\
& Reverse & TGGAATGGATGGATGGAGAT & 60 seconds \\
GAPDH Forward & GTCGGAGTCAACGGATTTGG & $\mathrm{N}^{\circ}$ cycles: 30 \\
& Reverse & GGGTGGAATCAATTGGACAT & Final extension step: $72^{\circ} \mathrm{C}$ \\
& & for 5 hours \\
\hline
\end{tabular}

ADSC at $24 \mathrm{hr}$

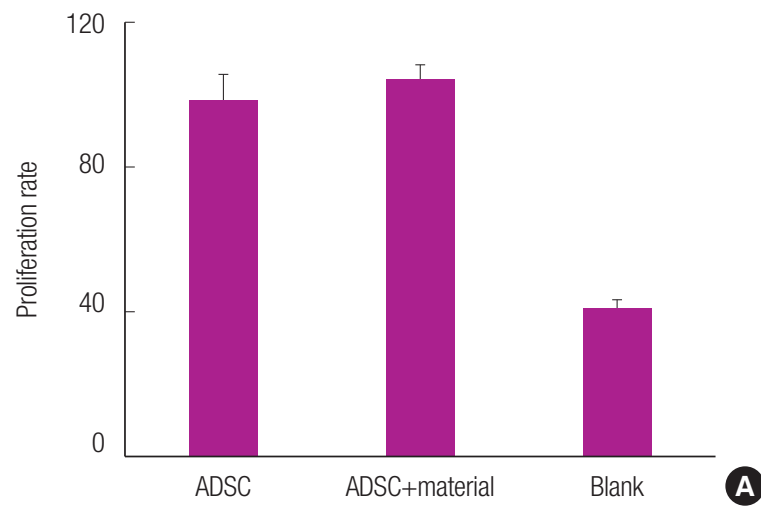

ADSC at 4 wk

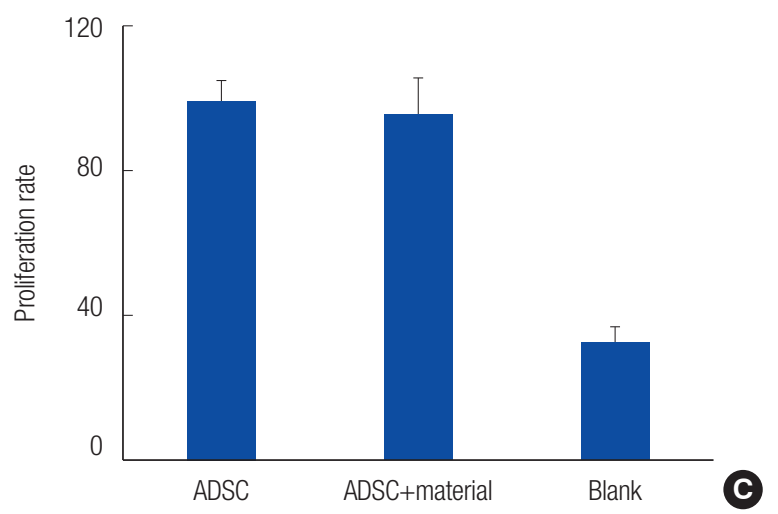

PCL/ $\beta$-TCP scaffold cell culture and the control cell culture 24 hours after the MTS assay. Similar results were observed at 4 weeks. Thus, the application of the $3 \mathrm{D}$ printed PCL/ $\beta-\mathrm{TCP}$ scaffold on MSCs was confirmed to be safe (Fig. 2).

\section{Comparison of osteogenic differentiation of various MSCs in the presence of 3D printed PCL/ $\beta$-TCP scaffold}

The obtained ADSCs and BMSCs were cultured in the 3D printed PCL/ $\beta$-TCP scaffold, and their levels of osteogenic differentiation were compared on days 1 and 10 and week 4 (Fig. 3). In the ALP assay, the osteogenic differentiation activity increased more in groups $\mathrm{M}$ and $\mathrm{D}$ than it did in group C. However, the osteogenic differentiation activity decreased more in group $\mathrm{M}$ than it did in group D (Fig. 4). The von Kossa staining performed at 4 weeks showed increased osteogenic differentiation activity in group $\mathrm{M}$ compared to that in group $\mathrm{C}$. The osteogenic differentiation activity increased even more in BMSCs than it did in group D (Fig. 5). Expression levels of ossification genes (COL I, OCN, and RUNX2) were compared using RTPCR. In ADSCs, the expression level of the RUNX2 gene at 4 weeks was slightly decreased; in BMSCs, the expression levels
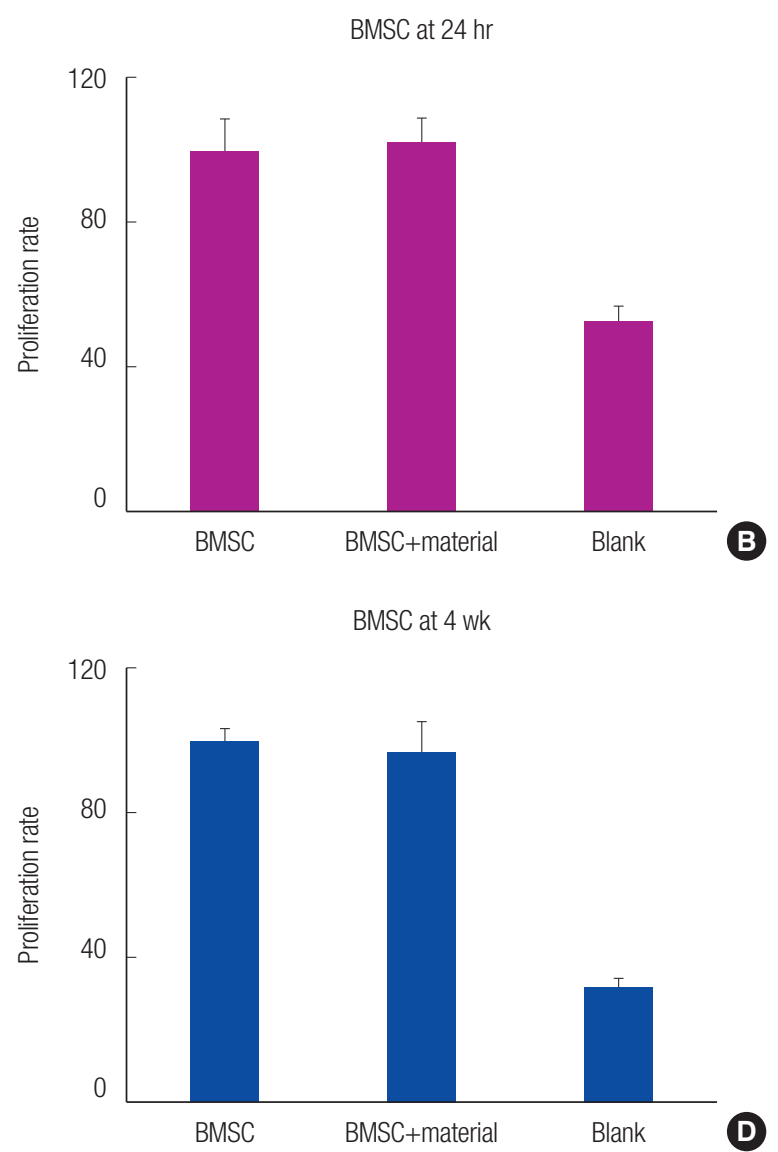

Fig. 2. Toxicity test results of three-dimensionally printed polycaprolactone/ $\beta$-tricalcium phosphate scaffold 24 hours (A, B) and 4 weeks (C, D) after cell culture. ADSC, adipose tissue-derived stem cell; BMSC, bone marrow-derived stem cell. 
of $O C N$ at days 1 and 10 were slightly decreased; and the expression level of the COL I gene at day 10 was slightly decreased. The gene expression levels in group $M$ were generally higher than those in group $\mathrm{C}$ except for some genes at specific time points. However, compared with the group D, the gene expression was slightly decreased at all time points. This suggests that the osteogenic differentiation in group $M$ was increased more than that in group $\mathrm{C}$, but decreased more than that in group D (Fig. 6).

Western blotting was performed to analyze the expression of ossification-related proteins. In ADSCs, the expression level of the RUNX2 protein at day 10 was slightly decreased in group $M$ compared to group C. Furthermore, in BMSCs, the expression levels of all proteins were decreased at day 1 , and the expression
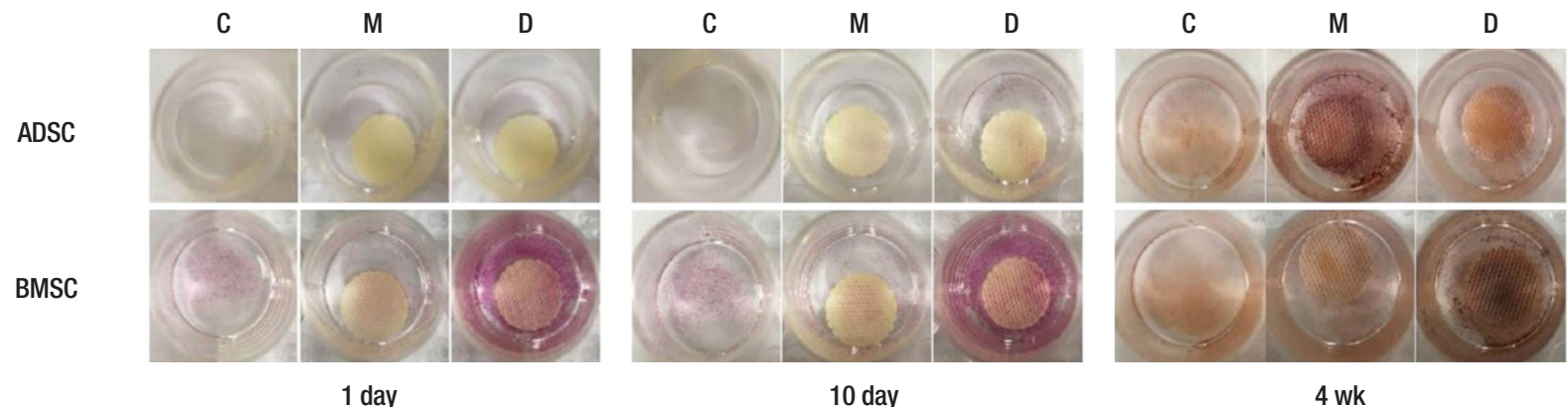

Fig. 3. Interaction between the three-dimensionally (3D) printed polycaprolactone (PCL)/ $\beta$-tricalcium phosphate (TCP) scaffold and cells. Alkaline phosphatase assay was performed 1 and 10 days after cell culture, followed by von Kossa staining at 4 weeks. Group C, only cultured cells; $\mathrm{M}$, cells cultured in the $3 \mathrm{D}$ printed PCL/ $\beta$-TCP scaffold; $\mathrm{D}$, cells cultured in the $3 \mathrm{D}$ printed PCL/ $\beta$-TCP scaffold with a bone differentiation medium; ADSC, adipose tissue-derived stem cell; BMSC, bone marrow-derived stem cell.
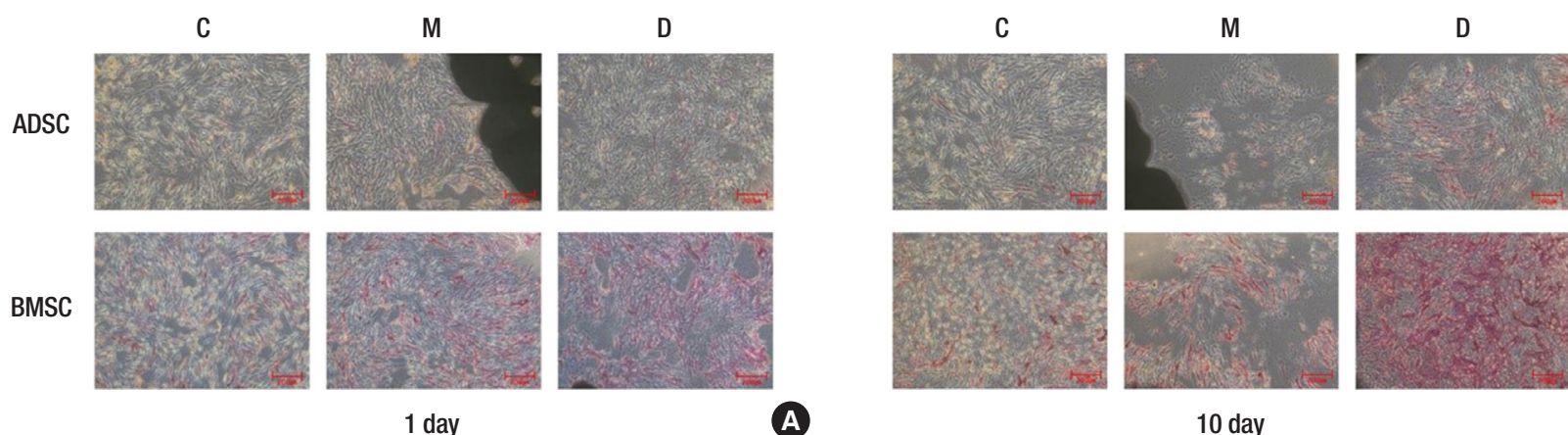

(A)

10 day

Fig. 4. Alkaline phosphatase assay of osteogenic differentiation. (A) Day $1, \times 5$ magnification. (B) Day $10, \times 5$ magnification. Group C, only cultured cells; $\mathrm{M}$, cells cultured in the three-dimensionally (3D) printed polycaprolactone (PCL)/ $\beta$-tricalcium phosphate (TCP) scaffold; D, cells cultured in the $3 \mathrm{D}$ printed PCL/ $\beta$-TCP scaffold with a bone differentiation medium; ADSC, adipose tissue-derived stem cell; BMSC, bone marrow-derived stem cell.
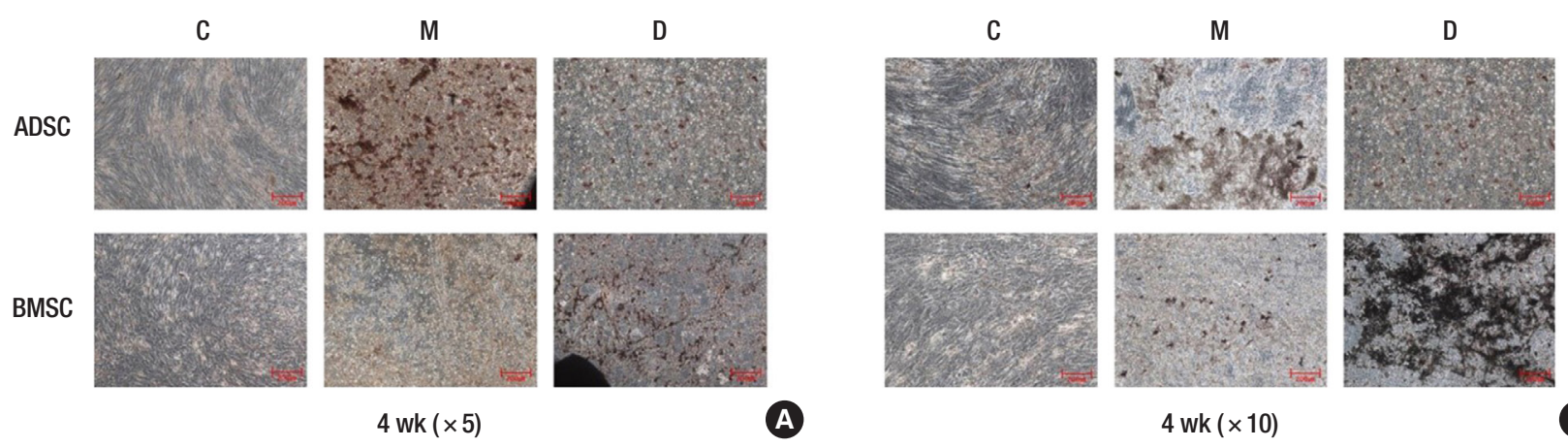

Fig. 5. von Kossa staining of osteogenic differentiation. (A) Week $4, \times 5$ magnification. (B) Week $4, \times 10$ magnification. Group C, only cultured cells; $\mathrm{M}$, cells cultured in the three-dimensionally (3D) printed polycaprolactone (PCL)/ $\beta$ - tricalcium phosphate (TCP) scaffold; D, cells cultured in the 3D printed PCL/ $\beta$-TCP scaffold with a bone differentiation medium; ADSC, adipose tissue-derived stem cell; BMSC, bone marrow-derived stem cell. 

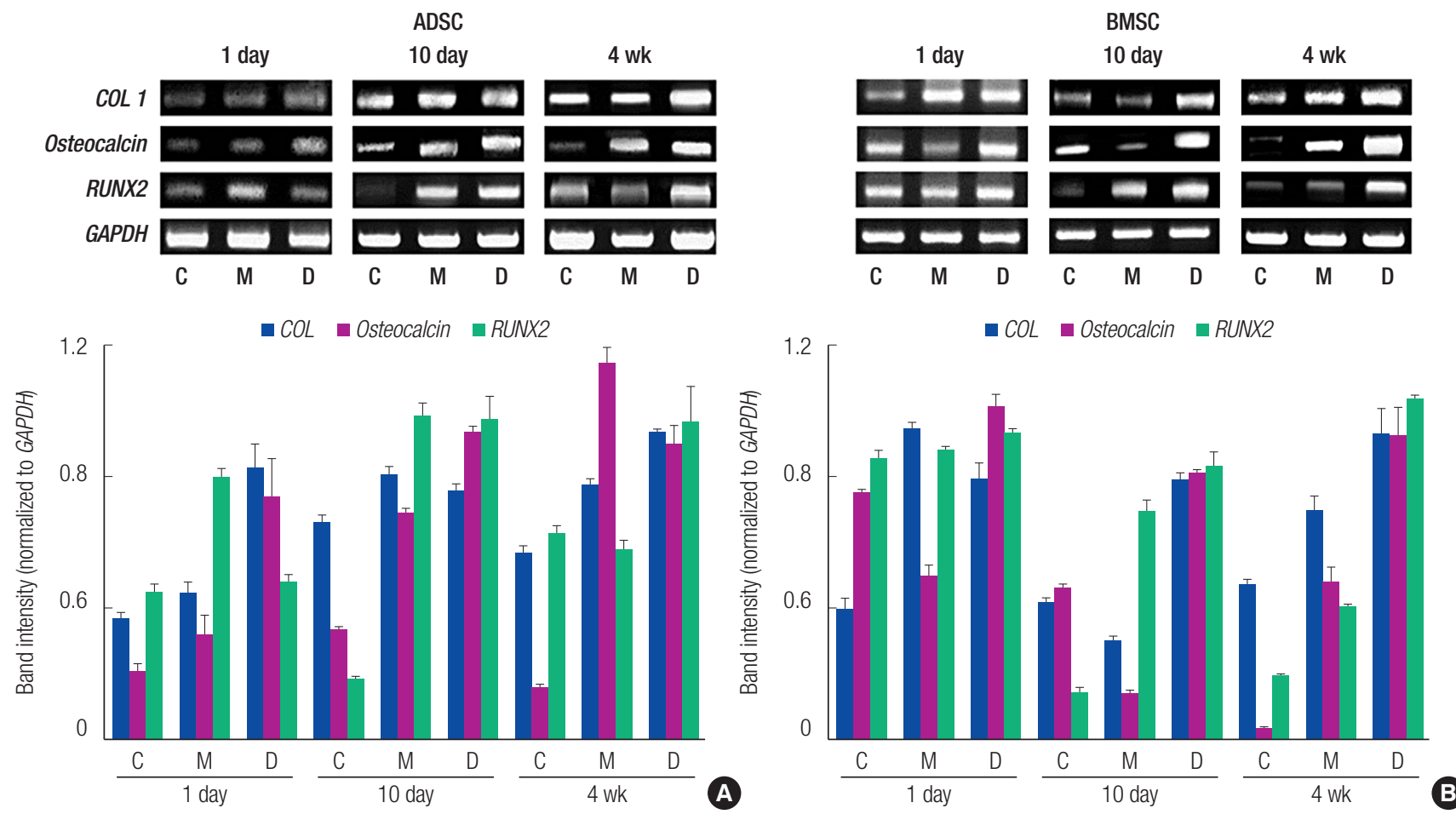

Fig. 6. Reverse transcription-polymerase chain reaction analysis of the expression levels of ossification genes (COL I, Osteocalcin, and RUNX2). (A) adipose tissue-derived stem cell (ADSC). (B) Bone marrow-derived stem cell (BMSC). Group C, only cultured cells; M, cells cultured in the three-dimensionally (3D) printed polycaprolactone (PCL)/ $\beta$-tricalcium phosphate (TCP) scaffold; D, cells cultured in the 3D printed PCL/ $\beta$-TCP scaffold with a bone differentiation medium; COL 1, type I collagen; RUNX2, runt-related transcription factor 2; GAPDH, glyceraldehyde-3-phosphate dehydrogenase.
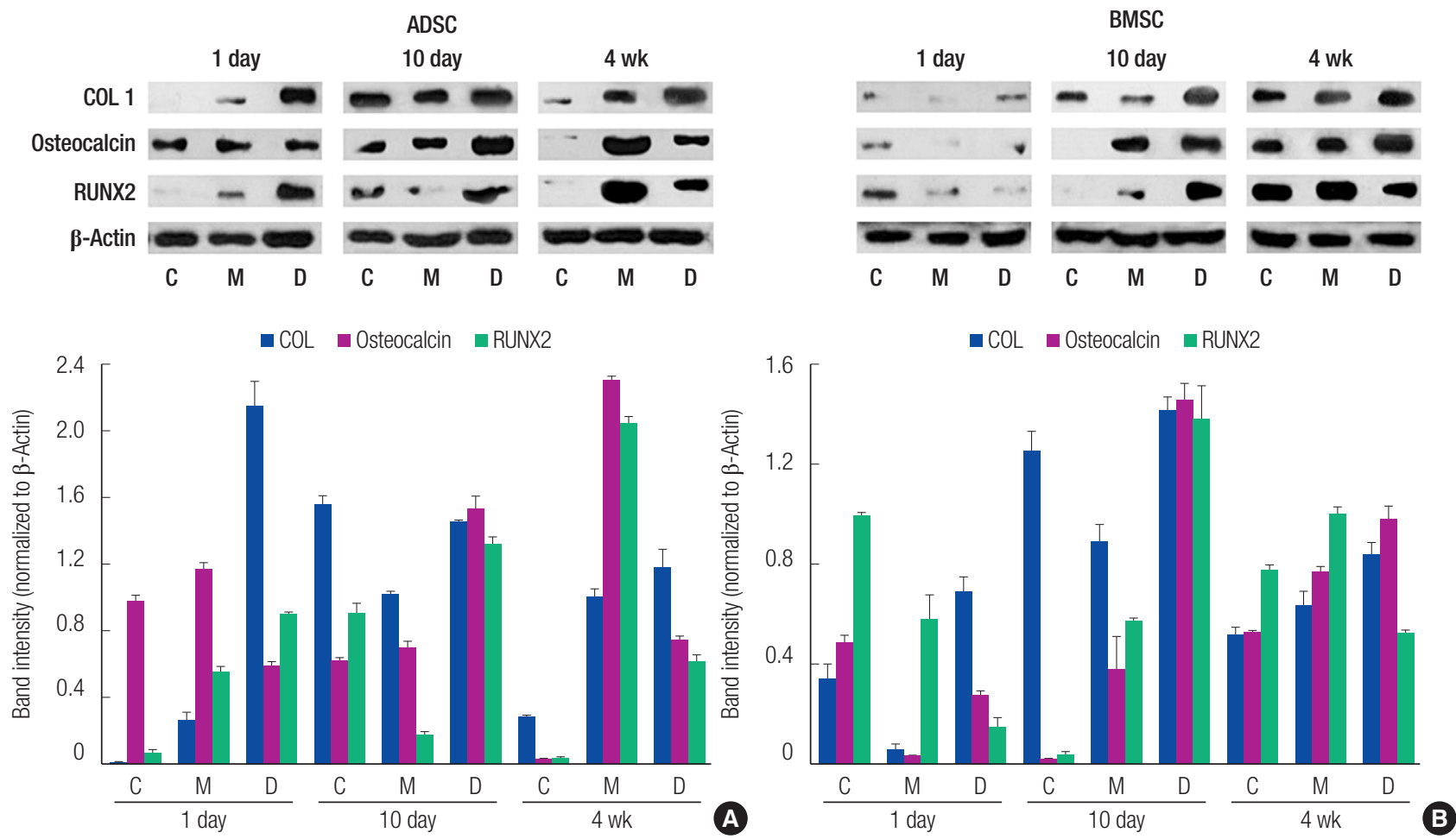

Fig. 7. Western blotting analysis of the expression of ossification-related proteins (COL I, Osteocalcin, and RUNX2). (A) Adipose-derived stem cell (ADSC). (B) Bone marrow-derived stem cell (BMSC). Group C, only cultured cells; $\mathrm{M}$, cells cultured in the three-dimensionally (3D) printed polycaprolactone (PCL)/ $\beta$-tricalcium phosphate (TCP) scaffold; $\mathrm{D}$, cells cultured in the $3 \mathrm{D}$ printed PCL/ $\beta$-TCP scaffold with a bone differentiation medium; COL 1, type I collagen; RUNX2, runt-related transcription factor 2. 
level of the COL I protein at day 10 was decreased in group $\mathrm{M}$ compared to group C. However, closer to 4 weeks, the protein expression levels in group $\mathrm{M}$ were increased compared to group C. This observation confirmed that osteogenic differentiation increased more in group $\mathrm{M}$ than it did in group C (Fig. 7).

\section{DISCUSSION}

The bone tissue engineering process consists of four main components: cells, scaffold, growth factors, and mechanics. Numerous studies have been conducted on bone tissue engineering and scaffold materials in the last two decades to identify the appropriate $3 \mathrm{D}$ environment that would increase the osteogenic efficiency of MSCs. The basic requirements for scaffold materials are biodegradability, biocompatibility, mechanical strength, 3D structure, and porous architecture with interconnected channels [10].

Numerous studies have confirmed that traditional scaffolds are adequate materials for bone tissue engineering. However, it is difficult to evenly distribute porogens of the same size as it is impossible to create uniform pore sizes, and the interconnections between pores are not complete. It is also difficult to form a structure with the desired form because the thickness and length of the pore wall and edges are determined by the solvent evaporation rate. Furthermore, any remaining organic solvents could have toxic effects on the body or the seeded cells and should be completely removed before clinical use of scaffolds [11].

Accordingly, numerous research studies have attempted to develop fabricating complexly shaped scaffolds using advanced techniques called solid free-form fabrication (SFF) or rapid prototyping (RP). One of the advantages of the SFF technique is that a customized architecture with compositional variations desired by the producer and of the same form can be reproduced. Moreover, in the production of biomimetic structures, SFF technology considers various scaffold designs and material compositions to produce a material architecture (size, shape, interconnectivity, branching, geometry, and orientation) tailored to the researcher's needs. Furthermore, it enables the manipulation of mechanical properties, biological effects, and degradation kinetics of scaffolds. The RP technique uses automated, integrated imaging technique to produce customized scaffolds of specific sizes or shapes that are tailored to each patient's needs. Commonly used SFF techniques include SLS, 3D printing, extrusion technology-based systems, solid grounding curing, and SLA [12], which have the following characteristics.

SLS increases the temperature using laser energy and fuses powder to produce a scaffold. It requires expensive equipment and traps powder, which is difficult to remove. SLA uses an ultraviolet (UV) laser to polymerize photopolymerizable liquid polymer materials. However, the number of photopolymerizable biomaterials is limited, and shrinkage and deformation can occur after UV curing. Extrusion technology-based systems, also called droplet deposition, include fused deposition modeling, 3D plotting, multi-phase jet solidification, and precise extrusion manufacturing. These methods involve the direct heating and pumping of filament materials on a built platform through a nozzle according to a programmed pathway. Some of the drawbacks of this technique are that its use is limited to thermoplastic materials that have good melting viscosity and it is difficult to encapsulate cells in the scaffold produced using this method [13].

The 3D printing technology used in this study was developed at the MIT, and is currently the most researched SFF technique in the fields of tissue engineering and drug-delivery applications [12]. The 3D printing technology uses a powder as the basic material and involves a layered printing process using adhesive bonding according to a sliced cross-sectional computerassisted design. Each powder layer becomes selectively fused by the ink-jet printing of binder materials, and the desired scaffold is created as this step is repeated layer by layer. The removal of harmful organic solvents such as chloroform and methylene chloride, which are used as binders during this process, is difficult. It is also difficult to remove the excess powder trapped inside the small channel. However, 3D printing can theoretically use any materials that can be powdered and in practice can be used in any environment as it is commonly used, and easy to perform [13].

It has already been proven that the combination of a scaffold and MSCs more effectively enhances osteogenic differentiation and, subsequently, bone regeneration than a scaffold alone does. In a study that analyzed the effects of urine-derived stem cells (UDSCs) and $\beta$-TCP on femoral bone defects of rats, bone regeneration was more effective when UDSCs and $\beta$-TCP were used in conjunction than when $\beta$-TCP was used alone [14]. In a study that treated mice having $4 \mathrm{~mm}$ calvarial defects with ADSCs and wet-spun starch-PCL (SPCL), better bone regeneration was achieved when SPCL and ADSCs were used together than when SPCL was used independently [15].

A study on the effects of the PCL scaffold on the osteogenic differentiation of umbilical cord-, bone marrow-, and adipose tissue-derived MSCs reported that PCL nanofibers enhance the osteogenic differentiation of human MSCs and, subsequently, MSC-based bone tissue repair. The PCL nanofiber scaffold induces the adhesion and proliferation of human MSCs to maintain the survival rate of human MSCS and accelerate their pro- 
liferation. Osteogenic differentiation potency could be significantly increased by culturing these human MSCs in the PCL nanofiber scaffold. In addition, of the three types of MSCs, BMSCs had the highest osteogenic differentiation potency, and the PCL nanofiber scaffold consistently activates the Wnt/ $\beta$-catenin and Smad3 signaling pathways to enhance the osteogenesis of human MSCs [6].

PCL, which is commonly used as a scaffold material, has a repetitive molecular structure of PCL homopolymers consisting of five nonpolar methylene groups and a single relatively polar ester group. While it has a high olefinic content and unique features similar to those of polyolefin, its polymers become biodegradable when fused with hydrolytically unstable aliphatic-ester. Homopolymer degradation takes approximately 2 years. Bioabsorption of these homopolymers can be accelerated by combining them with copolymers [16]. PCL is one such biodegradable polyester material that has been approved by the Food and Drug Administration for its good biocompatibility and low immunogenicity. It is also one of the most widely used biomaterials in various biomedical fields because of its low melting point $\left(58^{\circ} \mathrm{C}-63^{\circ} \mathrm{C}\right)$, easy handling, and low cost. However, it lacks cell adhesion owing to low hydrophilicity and must be coated with natural polymers such as collagen or combined with ceramics such as TCP to overcome its highly ductile and insufficiently rigid nature. TCP has strong osteoconductivity, high biocompatibility, and is degraded in vivo as it has a similar chemical composition to that of natural bone. Similar to PCL, it can also be used in various forms such as porous blocks, granules, and a powder [7].

The PCL/TCP scaffold effectively promotes osteoblast proliferation, differentiation, mineralized tissue formation and bone tissue ingrowth, and has degradation behavior, and is bioactive. In addition, a recent study reported that the PCL/TCP scaffold is involved in the delivery system of bone morphogenetic protein-2 and platelet-rich plasma and that it has sufficient physical properties to withstand the wound contraction force and load bearing application following in vivo implantation. Furthermore, the slow degradation induced by the scaffold provides sufficient time and space for new bone growth. Therefore, the PCL/TCP scaffold could be used as a safe and effective bone substitute for promoting osteogenic differentiation [17].

In the present study, the cells cultured in the 3D printed PCL/ $\beta$-TCP scaffold showed reduced osteogenic differentiation compared to cells that were cultured on the scaffold with a bone differentiation medium and showed increased osteogenic differentiation compared to that of the control group. Our findings demonstrate that osteogenic differentiation occurs as a result of the interactions between the PCL/ $\beta$-TCP scaffold and cells, and that the $3 \mathrm{D}$ printed PCL $/ \beta$-TCP scaffold alone promoted osteogenic differentiation. Furthermore, our findings provide evidence that the $3 \mathrm{D}$ printed PCL/ $\beta$-TCP scaffold is an effective synthetic biomaterial for promoting bone regeneration in bone tissue engineering.

\section{CONFLICT OF INTEREST}

No potential conflict of interest relevant to this article was reported.

\section{REFERENCES}

1. Liao HT, Lee MY, Tsai WW, Wang HC, Lu WC. Osteogenesis of adipose-derived stem cells on polycaprolactone- $\beta$-tricalcium phosphate scaffold fabricated via selective laser sintering and surface coating with collagen type I. J Tissue Eng Regen Med 2016;10:E337-53.

2. Barry FP, Murphy JM. Mesenchymal stem cells: clinical applications and biological characterization. Int J Biochem Cell Biol 2004;36:568-84.

3. Rose FR, Cyster LA, Grant DM, Scotchford CA, Howdle SM, Shakesheff KM. In vitro assessment of cell penetration into porous hydroxyapatite scaffolds with a central aligned channel. Biomaterials 2004;25:5507-14.

4. Sachlos E, Czernuszka JT. Making tissue engineering scaffolds work. Review: the application of solid freeform fabrication technology to the production of tissue engineering scaffolds. Eur Cell Mater 2003;5:29-39.

5. Arafat MT, Lam CX, Ekaputra AK, Wong SY, Li X, Gibson I. Biomimetic composite coating on rapid prototyped scaffolds for bone tissue engineering. Acta Biomater 2011;7:809-20.

6. Xue R, Qian Y, Li L, Yao G, Yang L, Sun Y. Polycaprolactone nanofiber scaffold enhances the osteogenic differentiation potency of various human tissue-derived mesenchymal stem cells. Stem Cell Res Ther 2017;8:148.

7. Matsuno T, Nakamura T, Kuremoto K, Notazawa S, Nakahara T, Hashimoto Y, et al. Development of beta-tricalcium phosphate/collagen sponge composite for bone regeneration. Dent Mater J 2006;25:138-44.

8. Choi D, Kumta PN. Mechano-chemical synthesis and characterization of nanostructured $\beta$-TCP powder. Mater Sci Eng C 2007;27:377-81.

9. Minoda R, Hayashida M, Masuda M, Yumoto E. Preliminary experience with beta-tricalcium phosphate for use in mastoid cavity obliteration after mastoidectomy. Otol Neurotol 2007;28: 1018-21.

10. Burg KJ, Porter S, Kellam JF. Biomaterial developments for 
bone tissue engineering. Biomaterials 2000;21:2347-59.

11. Yang S, Leong KF, Du Z, Chua CK. The design of scaffolds for use in tissue engineering. Part I: traditional factors. Tissue Eng 2001;7:679-89.

12. Hutmacher DW, Sittinger M, Risbud MV. Scaffold-based tissue engineering: rationale for computer-aided design and solid free-form fabrication systems. Trends Biotechnol 2004;22:35462.

13. Yang S, Leong KF, Du Z, Chua CK. The design of scaffolds for use in tissue engineering. Part II: rapid prototyping techniques. Tissue Eng 2002;8:1-11.

14. Guan J, Zhang J, Li H, Zhu Z, Guo S, Niu X, et al. Human urine derived stem cells in combination with $\beta$-TCP can be applied for bone regeneration. PLoS One 2015;10:e0125253.

15. Carvalho PP, Leonor IB, Smith BJ, Dias IR, Reis RL, Gimble JM, et al. Undifferentiated human adipose-derived stromal/ stem cells loaded onto wet-spun starch-polycaprolactone scaffolds enhance bone regeneration: nude mice calvarial defect in vivo study. J Biomed Mater Res A 2014;102:3102-11.

16. Middleton JC, Tipton AJ. Synthetic biodegradable polymers as orthopedic devices. Biomaterials 2000;21:2335-46.

17. Kim SJ, Kim MR, Oh JS, Han I, Shin SW. Effects of polycaprolactone-tricalcium phosphate, recombinant human bone morphogenetic protein-2 and dog mesenchymal stem cells on bone formation: pilot study in dogs. Yonsei Med J 2009;50:825-31. 